\title{
Estudo biomecânico comparando a utilização de placas bloqueadas híbridas na artrodese dorsal e medial do carpo de cães
}

\author{
[Biomechanical study comparing the utilization of hybrid locking plates on dorsal and medial \\ carpal arthrodesis in dogs] \\ L.M. Oliveira ${ }^{1}$, E.A. Tudury ${ }^{2}$, D.V.F. Lucena ${ }^{3}$, L.A.V.S. Costa ${ }^{2}$, B.M. Araújo ${ }^{4}$, \\ P.H.N. Cardoso ${ }^{5}$, N.C. Olivier ${ }^{6}$, D. Baraúna Júnior ${ }^{6}$ \\ ${ }^{1}$ Universidade Estadual da Região Tocantina do Maranhão - Imperatriz, MA \\ ${ }^{2}$ Universidade Federal Rural de Pernambuco - Recife, PE \\ ${ }^{3}$ Aluno de pós-graduação - Universidade Federal Rural de Pernambuco - Recife, PE \\ ${ }^{4}$ Universidade Federal do Piauí - Teresina, PI \\ ${ }^{5}$ Aluno de pós-graduação - Universidade Federal do Vale do São Francisco - Petrolina, PE \\ ${ }^{6}$ Universidade Federal do Vale do São Francisco - Petrolina, PE
}

\begin{abstract}
RESUMO
Objetivou-se com este trabalho comparar biomecanicamente os posicionamentos dorsal e medial da placa bloqueada na artrodese do carpo em cães. Para isso, foram utilizados sete pares de membros torácicos de cães eutanasiados por razões não relacionadas ao estudo, em que sete membros foram estabilizados com placa dorsal, e seus respectivos pares com placa em posicionamento medial. Os membros com as articulações radiocarpometacarpianas estabilizadas cirurgicamente foram submetidos a ensaio de compressão axial em máquina universal de ensaios de materiais, e os dados de limite de elasticidade, rigidez estrutural, carga máxima e carga no momento da fratura dos posicionamentos foram comparados por meio do teste de Student-Newman-Keuls, a um nível de significância de 5\%. Todos os membros foram fraturados no terço distal do terceiro metacarpo; nos membros com placa dorsal, a fratura ocorreu distal ao orifício distal e, nos membros com placa medial, a fratura ocorreu no último orifício dos metacarpos dois e três. Não houve dobra ou quebra de placas e parafusos e não houve diferença significativa entre os posicionamentos dorsal e medial dos implantes quanto aos parâmetros avaliados.
\end{abstract}

Palavras-chave: articulação, fratura, pan-artrodese, rigidez

\begin{abstract}
The aim of this study was to compare biomechanically the dorsal and medial positions of the locking plates on carpal arthrodesis in dogs. For this, seven pairs of thoracic limbs of dogs were used, in which seven limbs were stabilized with dorsal plate and seven limbs with plate in medial positioning. The members with the surgically stabilized radiocarpal-metacarpal joints were submitted to axial compression testing in a universal testing machine and the yield load, structural rigidity, maximum load and ultimate load of the positioning were compared by Student-Newman-Keuls test at a significance level of 5\%. All members fractured in the distal third of the third metacarpal, in the limbs with dorsal plate the fracture occurred distal to the distal hole and in the members with medial plate the fracture occurred in the last hole of the metacarpals two and three. There was no bending or broken implants. There was no significant biomechanical differencebetween the dorsal and medial positions of the implants.
\end{abstract}

Keywords: joint, fracture, panarthrodesis, rigidity

Recebido em 15 de agosto de 2017

Aceito em 9 de março de 2018

E-mail: oliveira.leovet@gmail.com 


\section{INTRODUÇÃO}

A pan-artrodese da articulação do carpo é uma cirurgia indicada em casos de hiperextensão radiocarpal irreversível ou no tratamento de transtornos irreversíveis que cursem com claudicação crônica (Bristow et al., 2015; Chaves et al., 2015; Shaughnessy et al., 2016).

Várias técnicas de artrodese do carpo são descritas em pequenos animais e todas se baseiam na remoção das superfícies articulares e na estabilização rígida da articulação radiocarpometacarpiana, objetivando a fusão óssea. A técnica mais indicada atualmente em pequenos animais é realizada com placa híbrida de compressão dinâmica colocada na face dorsal do carpo (DeCamp et al., 2016), entretanto a utilização de placas bloqueadas poderia oferecer mais estabilidade, já que estas apresentam maior resistência às forças responsáveis por falhas na estabilização (Cronier et al., 2010).

A face medial do carpo, de modo semelhante ao que ocorre na face palmar, também sofre tensão durante a sustentação do peso (Milgram et al., 2012; DeCamp et al., 2016); assim, a estabilização com placa por acesso medial poderia trazer vantagens, uma vez que há conversão de força tênsil de distração em força de compressão, minimizando as chances de falhas nos implantes e a ocorrência de fraturas (Guillou et al., 2012). Adicionalmente, nesse posicionamento, a placa oferece maior área de momento de inércia, tornando a fixação mais resistente (Guerrero e Montavon, 2005; Chao et al., 2012; Guillou et al., 2012).

A comparação da estabilidade proporcionada por essas técnicas é feita por meio de ensaios de compressão axial em que o membro, com as articulações do carpo cirurgicamente estabilizadas, é submetido a ensaios com cargas máximas predeterminadas (Guillou et al., 2012) ou até que haja falha de algum dos componentes do sistema osso/implante (Arnott et al., 2008; Rothstock et al., 2012; Burton et al., 2013). Apesar de haver trabalhos com avaliação biomecânica de diversos tipos de placas para artrodese (Viguier et al., 2001; Guerrero e Montavon, 2005; Rothstock et al., 2012; Bristow et al., 2015), não há trabalhos comparando a utilização de placas bloqueadas na rigidez e estabilidade da fixação. Nesse sentido, objetiva-se com este trabalho comparar biomecanicamente a artrodese do carpo pelas técnicas dorsal e medial com placas bloqueadas híbridas.

\section{MATERIAL E MÉTODOS}

Após aprovação do Comitê de Ética no Uso de Animais da Universidade Federal Rural de Pernambuco (Processo 23082.010338/2017-69), foram utilizados sete cadáveres de cães, machos e fêmeas, sem raça definida, de um a cinco anos de idade, pesando entre 20 e $30 \mathrm{~kg}$, que foram a óbito por razões não relacionadas ao projeto, oriundos do Centro de Vigilância Ambiental de Recife, PE. Após o óbito, os membros torácicos foram desarticulados no cotovelo, radiografados, e as imagens avaliadas quanto à ocorrência de osteopenia, bem como de quaisquer alterações ósseas. Caso algum membro apresentasse alteração radiográfica, os dois membros eram descartados.

Os sete membros direitos foram submetidos à pan-artrodese dorsal, com placa híbrida bloqueada 2,7/3,5 (Focus Produtos Ortopédicos, Brasil), e os sete membros esquerdos foram estabilizados com placa em posicionamento medial. As placas em aço inoxidável 316L ASTM F 138 foram confeccionadas exclusivamente para este experimento (Fig. 1).

Para a abordagem dorsal, foi feita incisão cutânea do terço médio do rádio à extremidade distal do terceiro metacarpo, seguida pela incisão da fáscia do antebraço medialmente ao músculo extensor digital comum e pela exposição dos grupos musculares e tendões extensores. Em seguida, a origem do músculo extensor longo do polegar foi elevada para preparação da superfície do terço distal do rádio onde a placa foi posicionada; a fáscia do carpo foi seccionada para liberação do tendão extensor digital comum, as inserções da cápsula articular no osso intermédio radial foram seccionadas $\mathrm{e}$, por último, o tendão do extensor carporradial foi seccionado na sua inserção no terceiro metacarpo (DeCamp et al., 2016).

Para abordagem medial, a pele foi incisionada do terço distal do rádio ao terço distal do segundo metacarpo, na face medial do membro, seguida pela incisão das fáscias do antebraço e do carpo e amputação do primeiro dígito por meio de 
incisão cutânea elíptica e desarticulação carpometacarpiana (DeCamp et al., 2016).

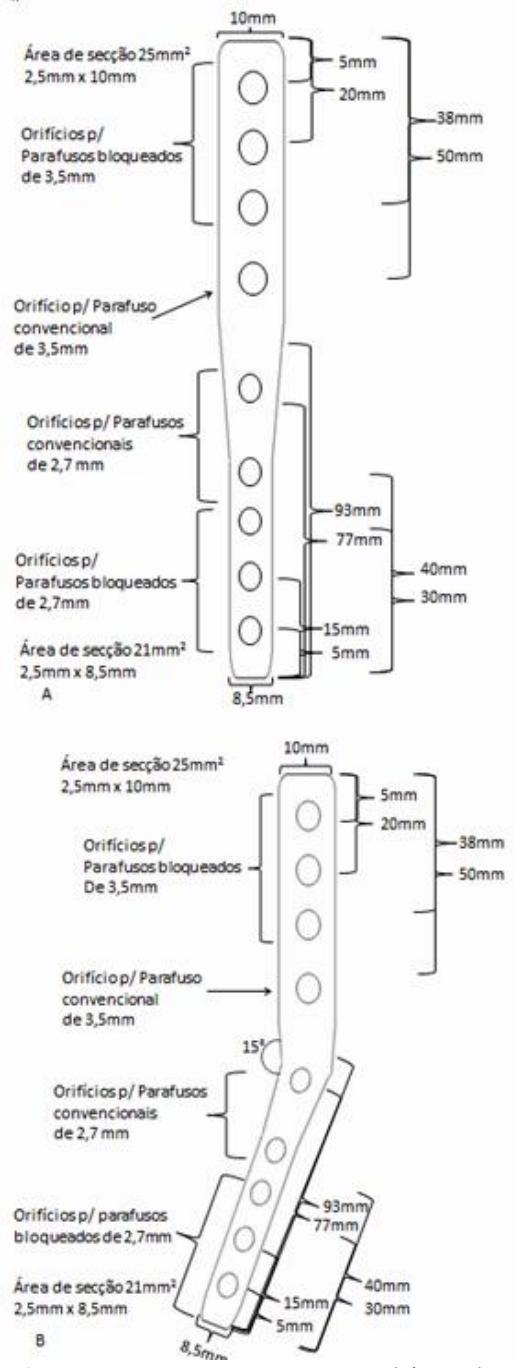

Figura 1. Representação esquemática de placas híbridas bloqueadas 2,7/3,5 para pan-artrodese do carpo. A- Placa para artrodese dorsal. BPlaca para artrodese medial.

A fixação das placas seguiu a mesma sequência nas duas abordagens. O primeiro parafuso foi colocado no orifício proximal do metacarpo, o segundo parafuso colocado no orifício distal do metacarpo, seguidos pelos demais parafusos do metacarpo e pelo parafuso no osso intermédio radial; o primeiro parafuso do rádio foi colocado no orifício distal e, em seguida, os demais. Nos membros com placa em posicionamento medial, os parafusos dos metacarpos foram fixados no segundo e terceiro metacarpianos. Foram utilizados três parafusos bloqueados (com haste central de $2,8 \mathrm{~mm}$ e profundidade da rosca de $0,35 \mathrm{~mm}$ ) e um não bloqueado (com haste central de $2,5 \mathrm{~mm}$ e profundidade da rosca de $0,5 \mathrm{~mm}$ ) de $3,5 \mathrm{~mm}$ no rádio, um parafuso não bloqueado de $2,7 \mathrm{~mm}$ (com haste central de $2,0 \mathrm{~mm}$ e profundidade da rosca de $0,35 \mathrm{~mm}$ ) no carpo radial e outro igual no orifício proximal do terceiro metacarpo, seguido de três parafusos bloqueados de $2,7 \mathrm{~mm}$ (com haste central de $2,0 \mathrm{~mm}$ e profundidade da rosca de $0,35 \mathrm{~mm}$ ) deste último osso, de modo que a placa proporcionasse mais de $60 \%$ de cobertura do metacarpo. As placas foram moldadas a um ângulo de $15^{\circ}$. Os parafusos bloqueados eram autoatarraxantes e utilizou-se guia de broca fixada com rosca nos orifícios da placa para perfurar os ossos, enquanto para os parafusos corticais, utilizou-se macho para a criação de rosca.

Optou-se por não serem removidas as cartilagens articulares devido à possibilidade de que o desgaste ósseo não ocorresse de modo idêntico em todos os membros, o que seria motivo de viés na comparação entre grupos (Arnott et al, 2008; Rothstock et al., 2012). Após a colocação das placas, o terço proximal do rádio e o da ulna foram expostos e centralmente fixados com pinos lisos de $2,5 \mathrm{~mm}$ a um cilindro de PVC de $10 \mathrm{~cm}$ de comprimento, que foi posteriormente preenchido com polimetilmetacrilato para 0 acoplamento na máquina de ensaios mecânicos.

Após a estabilização, as radiografias foram repetidas e, nos casos em que houve mau posicionamento dos implantes ou fratura, o membro e seu respectivo par foram excluídos do experimento. Os membros selecionados foram congelados a $-20^{\circ} \mathrm{C}$ até o dia dos ensaios mecânicos.

No dia anterior aos ensaios, os membros foram imersos em solução fisiológica e permaneceram em temperatura ambiente por $12 \mathrm{~h}$ para descongelamento. Em seguida, foram levados ao Laboratório de Ensaios Mecânicos do Colegiado de Engenharia Mecânica da Universidade Federal do Vale do São Francisco, em Juazeiro, BA. Os ensaios biomecânicos foram realizados na máquina universal de ensaios (MUE) (Máquina de ensaios EMIC DL - 5000/10000 Equipamentos e Sistemas de Ensaios Ltda., Brasil). A MUE possui uma interface com o software Tesc $^{\circledR}$ (Basil equipamentos científicos 
Ltda., Brasil), que permite a coleta e análise de dados referentes à velocidade, carga e deformação do material.

Cada membro foi submetido inicialmente a 10 ensaios de compressão em MUE (Fig. 2) para pré-condicionamento, onde foi fornecida uma pré-carga de $50 \mathrm{~N}$. Em seguida, foi realizada compressão a uma velocidade de $10 \mathrm{~mm} / \mathrm{min}$ até a carga máxima de $180 \mathrm{~N}$. Após essa etapa, o membro foi submetido a um ensaio destrutivo com mesma pré-carga, mas sem limite de força, até que houvesse fratura, caracterizada pelo declínio súbito da curva força x deformação. A partir deste último ensaio, foram registrados os valores de limite de elasticidade (LE), carga máxima $(\mathrm{CM})$, carga no momento da fratura $(\mathrm{CF})$ e rigidez estrutural (RE). Imediatamente após os ensaios, o ângulo de flexão de cada placa foi aferido com goniômetro para comparação com o ângulo inicial.

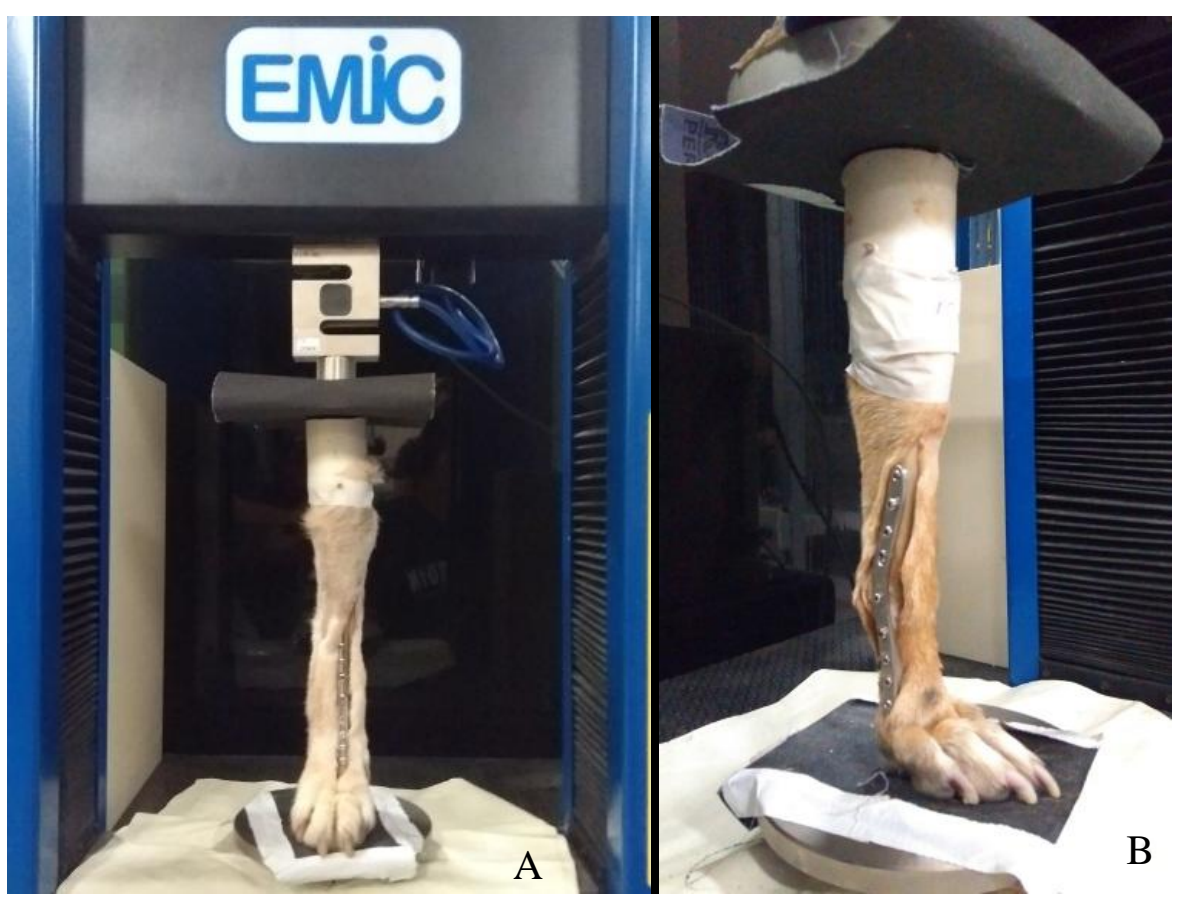

Figura 2. Membros estabilizados com placa bloqueada híbrida 2,7/3,5 em posicionamento dorsal (A) e em posicionamento medial (B), acoplados à máquina universal de ensaios mecânicos EMIC DL durante ensaio de compressão axial.

Inicialmente, em cada ensaio destrutivo, foi identificada a zona elástica (ZE), definida como o intervalo com alto coeficiente de determinação $\left(r^{2}>0,98\right)$ entre força e deformação na porção ascendente do gráfico. O primeiro e o último registro de força e deformação da $\mathrm{ZE}$ foram utilizados para o cálculo de RE ( $\Delta$ força/ $\Delta$ deformação) em $\mathrm{N} / \mathrm{mm}$. O primeiro registro de força $(\mathrm{N})$ após a $\mathrm{ZE}\left(\mathrm{r}^{2}<0,98\right)$ é o LE e marca o início da zona plástica. Também foram registradas, em Newtons, a $\mathrm{CM}$ atingida no ensaio e a CF.

Posteriormente aos ensaios, as radiografias foram repetidas antes e após a retirada das placas para avaliação dos implantes e ossos.
Os dados de limite de elasticidade, rigidez estrutural, carga máxima e carga no momento da fratura dos posicionamentos apresentaram distribuição normal no teste de Shapiro-Wilk e foram comparados por meio do teste de StudentNewman-Keuls, a um nível de significância de 5\% (BIOSTAT 5.0).

\section{RESULTADOS E DISCUSSÃO}

Em todos os ensaios destrutivos, os membros foram fraturados no orifício distal dos metacarpos (Fig. 3), exceto em dois com placa dorsal, em que a fratura ocorreu distal ao último orifício. Houve fratura nessa localização possivelmente porque a placa transfere a carga 
suportada pelo membro torácico para o terceiro metacarpo, quando se utiliza placa dorsal (Rothstock et al., 2012), ou para o segundo e terceiro metacarpos, quando se utiliza placa medial, o que torna o segmento distal desses ossos um ponto de concentração de tensão. Embora se reconheça que a redução da área de secção transversal óssea na altura dos orifícios torne essa região mais frágil (Gautier e Sommer, 2003), não há correlação entre o diâmetro dos parafusos e a ocorrência de fratura metacárpica (Denny e Barr, 1991; Whitelock et al., 1999; Wininger et al., 2007; Bristow et al., 2015).

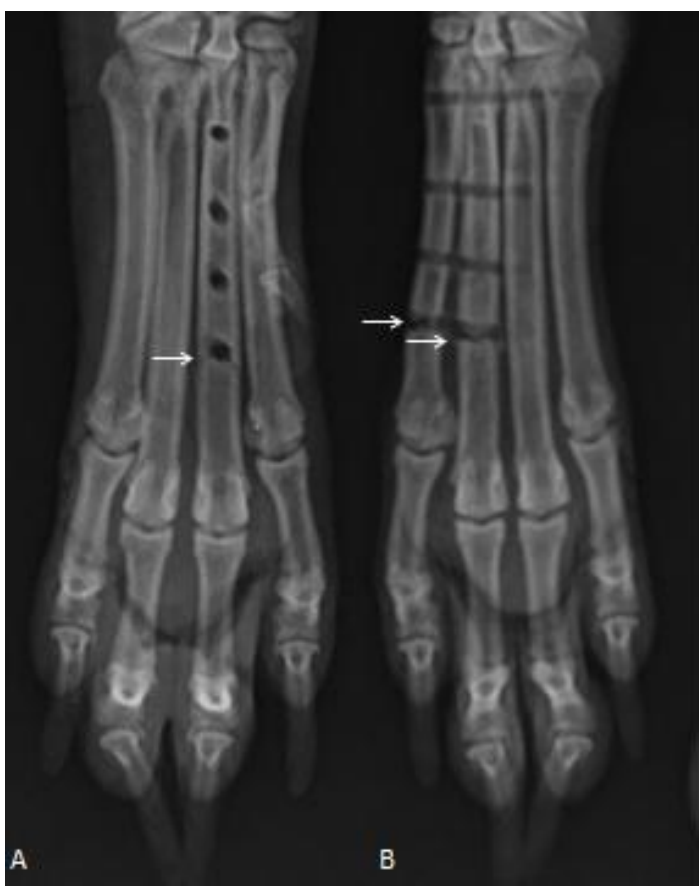

Figura 3. Radiografia realizada após a retirada das placas de artrodese dorsal (A) e medial (B). Notar que as fraturas (setas) ocorreram nos orifícios distais dos metacarpos. Nos dois posicionamentos, a placa foi fixada a cerca de $60 \%$ do terceiro metacarpo; sabe-se que, quando a placa cobre menos de $50 \%$ do metacarpo nas artrodeses dorsais, há um maior risco de fratura (Whitelock et al., 1999) e que, para a artrodese medial, cobertura inferior a $40 \%$ resulta em falha nos parafusos distais (Guerrero e Montavon, 2005).
Não houve diferença estatisticamente significante entre os posicionamentos dorsal e medial, apesar de as médias de LE, CM e CF apresentarem valores superiores no posicionamento medial (Tab. 1). De acordo com DeCamp et al. (2016), a face medial do carpo sofre tensão durante a extensão articular, de modo que a colocação da placa na face medial pode favorecer o compartilhamento da carga entre os implantes e os ossos, minimizando os efeitos da flexão cíclica. Essa vantagem não foi observada neste estudo possivelmente devido à resistência dos implantes utilizados, já que a área de secção das placas utilizadas $\left(25 \mathrm{~mm}^{2}\right.$ no rádio e $21,25 \mathrm{~mm}^{2}$ no metacarpo) foi maior que os $20 \mathrm{~mm}^{2}$ necessários para estabilização de fraturas do rádio e os $4 \mathrm{~mm}^{2}$ para o metacarpo (Slocum e Slocum, 1998), o que certamente conferiu resistência além do necessário para a estabilização do carpo.

Sendo a flexão o principal movimento do carpo, a placa em posicionamento medial proporciona uma maior área de momento de inércia $\left(208,33 \mathrm{~mm}^{4}\right)$ quando comparada ao posicionamento dorsal $\left(13,02 \mathrm{~mm}^{4}\right)$, o que poderia promover maior resistência (Guerrero e Montavon, 2005; Guillou et al., 2012; Chao et al., 2012). Entretanto, o movimento de lateralização da mão devido ao ângulo valgus do carpo durante a compressão axial (Milgram et al., 2012) pode ter compensado o ganho que o momento de inércia do posicionamento medial, por ventura, trouxesse, já que, para antagonizar esse movimento, a área momento de inércia é igual à do posicionamento dorsal, e isso pode ter suprimido a manifestação da vantagem do posicionamento medial.

A zona neutra (Gráf. 1), que corresponde à região da curva força $\mathrm{x}$ deformação onde $\mathrm{a}$ variação da deformação é maior que a variação da força, representa deformação de tecidos moles como a extensão das articulações dos dígitos e a compressão dos coxins (Viguier et al., 2001). Essa fase não apresenta dados úteis para comparação biomecânica nesse tipo de experimento. 
Tabela 1. Valores individuais, médias e desvio-padrão (DP) de limite de elasticidade (LE), carga máxima $(\mathrm{CM})$, carga no momento da fratura $(\mathrm{CF})$ e rigidez estrutural (RE) de pares de membros torácicos submetidos à artrodese do carpo com placa bloqueada híbrida 2,7/3,5 em posicionamento dorsal e medial

\begin{tabular}{ccccccccc} 
& \multicolumn{2}{c}{ LE (N) } & \multicolumn{2}{c}{ CM (N) } & \multicolumn{2}{c}{ CF (N) } & \multicolumn{2}{c}{ RE (N/mm) } \\
Par & Dorsal & Medial & Dorsal & Medial & Dorsal & Medial & Dorsal & Medial \\
\hline 1 & 873,3 & 1155,2 & 879,5 & 1219,3 & 838,5 & 1201,6 & 137,7 & 163,3 \\
2 & 1018,7 & 111,1 & 1035,3 & 1130,60 & 1017,4 & 1114,3 & 153,7 & 135,7 \\
3 & 820,8 & 1417,2 & 825,6 & 1417,2 & 811,3 & 1406,3 & 138,1 & 186,8 \\
4 & 1048,1 & 965,5 & 1066,5 & 992,8 & 1049,4 & 979,8 & 181,2 & 74,6 \\
5 & 1124,5 & 799,7 & 1127,9 & 805,16 & 1110,2 & 794,9 & 135,1 & 140,7 \\
6 & 1279,4 & 985,9 & 1280,7 & 985,98 & 1274,6 & 958,6 & 155,5 & 82,3 \\
7 & 1026,2 & 1340,1 & 1031,7 & 1363,3 & 1020,8 & 1344,9 & 174,6 & 166,3 \\
Média & 1027,31 & 1110,6 & 1035,3 & 1130,6 & 1017,4 & 1114,3 & 153,7 & 135,7 \\
DP & 141,3 & 200,6 & 140,4 & 203,5 & 146,5 & 203,6 & 17,0 & 39,4 \\
P= & & 0,4225 & & 0,3625 & & 0,3638 & & 0,3248 \\
\hline
\end{tabular}

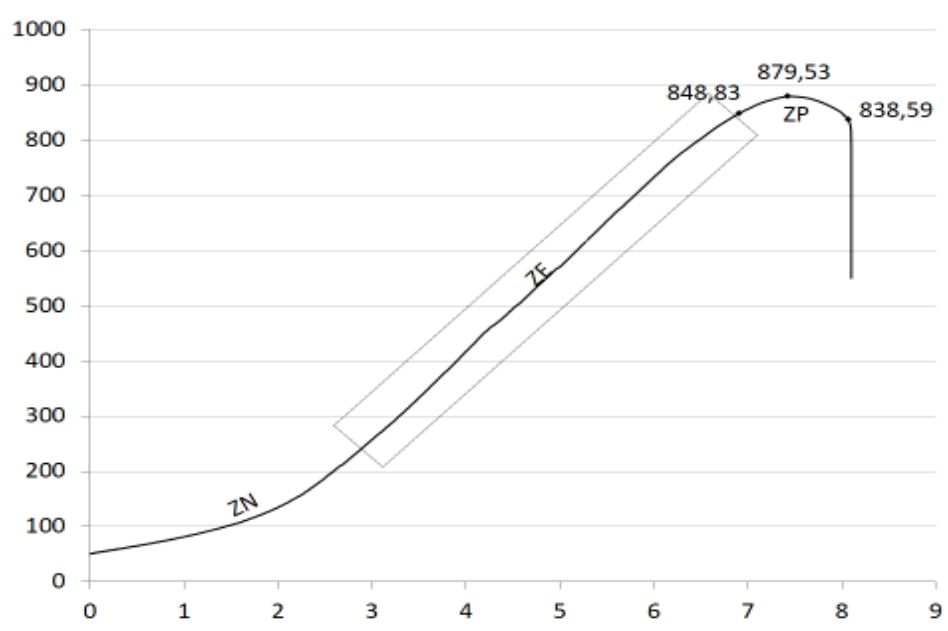

Gráfico 1. Gráfico força x deformação de compressão de artrodese carpal com placa bloqueada híbrida em posicionamento dorsal representando a zona neutra $(\mathrm{ZN})$, zona elástica $(\mathrm{ZE})$, limite de elasticidade de $848,83 \mathrm{~N}$, zona plástica (ZP), carga máxima de $879,53 \mathrm{~N}$ e carga no momento da fratura de $838,59 \mathrm{~N}$.

A zona elástica (ZE) foi identificada de modo bem característico em todos os ensaios (Gráf. 1). Nessa fase da compressão, caso o ensaio seja interrompido, a deformação produzida será revertida pela retirada da carga sobre o membro, de modo que quanto mais rígida for a estabilização, mais força será necessária para promover deformação e, neste intervalo, calculase a rigidez estrutural (Kaddick, 2015; Zdero et al., 2017).

Os valores de rigidez estrutural e carga no momento da fratura registrados foram inferiores ao descrito por Arnott et al. (2008), ao avaliarem o efeito da utilização de dois fios de Kirschner transarticulares cruzados na artrodese dorsal do carpo utilizando placa híbrida convencional 2,7/3,5, o que pode ter ocorrido porque as placas utilizadas não foram anguladas. A angulação da placa pode contribuir para a ocorrência de fratura na extremidade do metacarpo por aumentar o momento de força nesse ponto (Whitelock, 1999). Assim, em ensaios com placas não anguladas, onde há apenas compressão axial, o conjunto implante-osso apresenta mais resistência (Kaddick, 2015). Entretanto, é 
necessário angular a placa em $10-15^{\circ}$ para que o apoio do membro aconteça de modo semelhante ao fisiológico (DeCamp et al., 2016).

Não houve deformação em placas ou parafusos, o que permite supor que o limite de elasticidade (LE) registrado corresponde à porção distal do terceiro metacarpo e que o LE da placa não foi alcançado, resultado que classifica a fixação como estável (Frigg et al., 2007). O LE é a carga a partir da qual a deformação produzida não pode ser revertida após o alívio da carga (Kaddick, 2015) e é um parâmetro importante para comparação das propriedades mecânicas de diferentes placas, uma vez que a carga à qual a placa será submetida não deve ultrapassar este ponto para que o alinhamento entre os fragmentos se mantenha (Chao et al., 2012).

\section{CONCLUSÃO}

Apesar de o posicionamento medial do implante na pan-artrodese carpal ter apresentado médias superiores, a localização da placa híbrida bloqueada 2,7/3,5 não influenciou de forma significante na rigidez estrutural, no limite de elasticidade, na carga máxima e na carga no momento da fratura do conjunto placa-osso.

\section{REFERÊNCIAS}

ARNOTT, J.L.; BAILEY, R.; SHIELDS, A. et al. An in vitro comparison of a $2.7 / 3.5 \mathrm{~mm}$ hybrid plate alone and combined with crossed K-wires for canine pancarpal arthrodesis. Vet. Comp. Orthop. Traumatol., v.21, p.307-311, 2008.

BRISTOW, P.C.; MEESON, R.L.; THORNE, R.M. et al. Clinical comparison of the hybrid dynamic compression plate and the castless plate for pancarpal arthrodesis in 219 dogs. Vet. Surg., v.44, p.70-77, 2015.

BURTON, N.J., MILES, A.W.; POLLINTINE, P. Biomechanical comparison of a novel castless arthrodesis plate with -plate and cross pin techniques for canine partial carpal arthrodesis. Vet. Comp. Orthop. Traumatol., v.26, p.165-171, 2013.

CHAO, P.; LEWIS, D.D.; KOWALESKI, M.P. et al. Biomechanical concepts applicable to minimally invasive fracture repair in small animals. Vet. Clin. Small Anim. Pract., v.42, p.853-872, 2012.
CHAVES, R.O.; BECKMANN, D.V.; COPAT, B. et al. Poliartrite em cães - 27 casos (20072013). Ciênc. Rural, v.45, p.533-539, 2015.

CRONIER, P.; PIETU, G.; DUJARDIN, C. et al. The concept of locking plates. Orthop. Traumatol. Surg. Res., v.96, p.17-36, 2010.

DeCAMP, C.E.; JOHNSTON, S.A. et al. Piermattei, and Flo's Handbook of small animal orthopedics and fracture repair. 5.ed. St. Louis: Elsevier, 2016. p.389-433.

DENNY, H.R.; BARR, A.R.S. Partial and pancarpal arthrodesis in the dog: a review of 50 cases. J. Small Anim. Pract., v.32, p.329-334, 1991.

FRIGG, R.; FRENK, A.; WAGNER, M. Biomechanics of plate osteosynthesis Techn. Orthop., v.22, p.203-208, 2007.

GAUTIER, E.; SOMMER, C. Guidelines for the clinical application of the LCP. Injury, v.34, p.B63-B76, 2003.

GUERRERO, T.S.G.; MONTAVON, P.M. Medial plating for carpal panarthrodesis. Vet. Surg., v.34, p.153-158, 2005.

GUILLOU, R.P.; DEMIANIUK, R.M.; SINNOTT, M.T. et al. In vitro mechanical evaluation of a limited contact dynamic compression plate and hybrid carpal arthrodesis plate for canine pancarpal arthrodesis. Vet. Comp. Orthop. Traumatol., v.25, p.83-88, 2012.

KADDICK, C. Static, dynamic, and fatigue mechanical testing. In: SIMPSON, A. HAMISH, R.W. Experimental research methods in orthopedics and trauma. Stuttgart: Thieme, 2015. cap.6, p.42-48.

MILGRAM, J.; MILSHTEIN, T.; MEINER, Y. The role of the antebrachiocarpal ligaments in the prevention of hyperextension of the antebrachiocarpal joint. Vet. Surg., v.4, p.191199, 2012.

ROTHSTOCK, S.; KOWALESKI, M.P.; BOUDRIEAU, R.J. et al. Biomechanical and computational evaluation of two loading transfer concepts for pancarpal arthrodesis in dogs. Am. J. Vet. Res., v.73, p.1687-1695, 2012. 
SHAUGHNESSY, M.L.; SAMPLE, S.J.; ABICHT, C. et al. Clinical features and pathological joint changes in dogs with erosive immune-mediated polyarthritis: 13 cases (20042012). J. Am. Vet. Med. Assoc., v.249, p.11561164, 2016.

SLOCUM, B.; SLOCUM, T.D. General principles. In: BOJRAB, M.J.; WALDRON, D. R.; TOOMBS, J.P. Current techniques in small animal surgery. 4.ed. Jackson, WY: CRC Press, 1998. p.865-893.

VIGUIER, E.; ZNATY, D.; MEDELCI, M. et al. In vitro comparison between a DCP and external fixator for pancarpal arthrodesis in the dog. Equine Vet. J., v.33, p.32-35, 2001.
WHITELOCK, R.G.; DYCE, J.; HOULTON, J.E.F. Metacarpal fractures associated with pancarpal arthrodesis in dogs. Vet. Surg., v.28, p.25-30, 1999.

WININGER, F.A.; KAPATKIN, A.S.; RADIN, A. et al. Failure mode and bending moment of canine pancarpal arthrodesis constructs stabilized with two different implant systems. Vet. Surg., v.36, p.724-728, 2007.

ZDERO, R.; AZIZ, M.S.R.; NICAYENZI, B. Quasi-static stiffness and strength testing of whole bones and implants. ZDERO, R. In: Experimental methods in orthopaedic biomechanics. London: Academic Press, 2017. p.19-32. 